\title{
Three-column Internal Fixation System for Comminuted Fracture of the Tibial Plateau
}

\author{
Ali Mohammed El Gioushy, Galal Mansour Hegazy, and Ahmed Ashour El Sherief* \\ Department of Orthopedic Surgery, Faculty of Medicine, Al-Azhar University, Egypt \\ *Corresponding author: Ahmed Ashour El Sherief, Mobile: (+20) 01007627623
}

\begin{abstract}
Background: the tibia is the major weight-bearing bone of the lower leg. The proximal portion of the bone, the tibial plateau, forms the lower surface of the knee joint and it consists of two condyles - medial and lateral - separated by the tibial spines, The articular surface of the medial plateau is concave, whereas that of the lateral plateau is convex. The knee is a complex dynamic joint that is subjected to many forces during normal activities, so intact articular surface of tibial plateau is one of important factors to keep the geometry and alignment of the knee joint to act in harmony to perform its crucial function as a flexible weight bearing joint.

Objective: the aim was to describe the operative procedures and clinical outcomes of internal fixation of tibial plateau to treat comminuted fractures of the tibial plateau according a new three-column classification.

Conclusion: using anatomical locking plates on tibial plateau is an effective and safe tool to treat complex three-column fractures of tibial plateau and is more convenient than common plate.
\end{abstract}

Keywords: Internal Fixation System, Comminuted, Tibial Plateau

\section{INTRODUCTION}

Tibial plateau fractures are among the most challenging intra-articular fractures to treat, fracture characteristics range from simple to complex $^{(\mathbf{1})}$. Complex fractures of tibial plateau are considered a difficult aspect of trauma orthopedics, because these kinds of fractures are mostly smashed to pieces, accompanied by severe injuries of soft tissue and articular cartilage ${ }^{(2)}$.

The comminuted fractures of the tibial plateau are usually seen in pedestrians struck by vehicles and also in work related accidents. These fractures are described by Luo et $\boldsymbol{a l} .^{(3)}$ as "Three- column fracture" which is fracture' is defined as at least one independent articular fragment in each column. The most common three-column fracture is a traditional 'bicondylar fracture' (Schatzker Type V or Type VI) combined with a separate posterolateral articular fragment ${ }^{(4)}$.

In these type of fracture and according three-column concept, open reductionand internal fixation of each column separately is the gold standard ${ }^{(4)}$. Osteosynthesis is performed from standard anterolateral and posteromedial surgical approaches ${ }^{(2)}$.

Many shapes of plates are used in fixation such as straight plate, T-shaped plate, L-shaped plate, clovershaped plate, and angular stable locking plates ${ }^{(5-8)}$. But these plates could not match the special bony structure of the posterior column which had irregular anatomic form, and had obvious curvature at metaphysis,so, many difficulties appeared, such as spending more time to mold plates, cutting soft tissue by up-warping plates, fracture displacement after compression through mismatching plates ${ }^{(2,9,10)}$.

To solve these problems, three-column internal fixation system has designed with anatomical locking plates on tibial plateau and it could shorten the internal fixation process, reduce operation time, reduce operation injury and improve operation efficiency ${ }^{(2,11,12)}$.

\section{AIM OF THE WORK}

The aim of this work is to describe the operative procedures and clinical outcomes of internal fixation of tibial plateau to treat comminuted fractures of the tibial plateau according a new three-column classification.

\section{Classifications of the tibial plateau fracture}

There are numerous classification systems that have been proposed to describe tibial plateau fractures. It is about six anatomical classification schemes are established in clinical practice ${ }^{(\mathbf{1 3})}$. The classical ones are the OTA/AO and the Schatzker classification systems ${ }^{(\mathbf{1 4})}$. Recently, a new three-column classification approach was proposed by Luo et al. ${ }^{(3)}$ based on multiplanar CT images.

\section{a) Hohl classification}

The Hohl classification was the first widely accepted description of tibial plateau fractures, classifying these fractures into displaced and undisplaced types. Under the displaced category, he recognized local compression, split compression, total condyle depression, and comminuted fractures ${ }^{(\mathbf{1 5})}$.

\section{b) Moore classification:}

Moore expanded upon Hohl's concepts, taking into account higher-energy injuries and resultant knee instability. His classification of fracture-subluxation of the knee is divided into five types. Type I is a split fracture of the medial tibial plateau in the coronal plane; Type II is an entire condyle fracture in which the fracture line begins in the opposite compartment and extends across the tibial eminence; Type III is a rim avulsion fracture (these fractures are associated with a high rate of associated neurovascular injury); Type IV is another type of rim fracture, a rim compression injury that is usually associated with some type of contralateral ligamentous injury; and Type $\mathrm{V}$ is a four-part fracture in which the tibial eminence is separated from the tibial condyles and the tibial shaft ${ }^{(15)}$. 


\section{c) Schatzker classification:}

In 1979, Schatzker et al $^{\left({ }^{(16)}\right.}$, proposed what has become the most widely accepted classification for tibial plateau fractures. Amalgamating many of the fractures of previous classification schemes, this classification groups plateau fractures into six basic types starting with the lateral condyle and moving medially:

- Type I: (split fracture) is a pure cleavage fracture of the lateral tibial plateau that results in a wedge-shaped fracture fragment.

- Type II: (split-depression) is a cleavage fracture of the lateral tibial plateau in which the remaining articular surface is depressed into the metaphysis.

- Type III: is a pure central depression fracture of the lateral tibial plateau with an intact osseous rim.

- Type IV: involves the medial tibial plateau and is divided into two subtypes: Type A, which is a split fracture and Type B, a depression fracture. Either type may be combined with a tibial spine fracture.

- Type $\mathbf{V}$ : is a bicondylar fracture in which the fracture line often forms an inverted Y; the metaphysis and diaphysis remain intact.

- Type VI: is a tibial plateau fracture in which there is dissociation between the metaphysis and the diaphysis; these fractures may have varying degrees of comminution of one or both tibial condyles and the articular surface.

The advantages of this classification are that it incorporates topographic and morphologic characteristics, pathophysiologic factors, and treatment. The topographic significance of the medial tibial plateau has been recognized in this classification. However the disadvantages of that classification are that some fractures still remained unclassified and in addition, fracturedislocations described by Moore have not been included ${ }^{(17)}$.

\section{d) $\mathrm{AO} / \mathrm{OTA}$ classification}

In the Orthopedic Trauma Association (OTA) classification, which is based on the Association for the Study of Internal Fixation (AO/ASIF) classification, the proximal tibia is denoted as segment and is divided into three main categories. Type A fractures are extra-articular. Type B fractures are partial articular and are subdivided into three main categories: B1 are pure splits, B2 are pure depression, and $\mathrm{B} 3$ are split-depression. Type $\mathrm{C}$ fractures are complete articular fractures and are also subdivided into three subtypes: Type 1 is articular and metaphyseal simple, Type 2 is articular simple and metaphyseal multifragmentary, and Type 3 is articular multifragmentary (17).

\section{e) The three-column classification:}

Since existing classification systems could not describe posterior tibial plateau fracture well, Luo $\boldsymbol{e t}$ al. ${ }^{(3)}$ proposed three-column classification of tibial plateau fracture on basis of CT with application of spiral
CT, dividing tibial plateau fracture into medial column, lateral column and posterior column. This method, helping orthopedists understand fracture classification well, is a valuable classification method of tibial plateau fracture and can help to guide choices of surgical approach and internal fixation methods, thus significantly improving diagnosis and treatment effect of posterior tibial plateau fracture ${ }^{(3)}$.

\section{Diagnosis of the Tibial Plateau Fracture}

\section{* Clinical assessment: \\ $>$ History}

To achieve an accurate diagnosis, carefully asking the medical history to help doctors in time understand knee flexion or extension status, intensity and direction of violence at the time of injury, thus having a preliminary judgment for classification of fracture $^{(\mathbf{1 8 )}}$. The mechanism of injury provides clues to the fracture pattern and should direct the necessary degree of vigilance for associated injuries, i.e., Split lateral plateau fractures typically result from low-energy forces from falls and twisting injuries, and the risk of associated neurovascular injury or compartment syndrome is very low ${ }^{(\mathbf{1 8})}$.

\section{$>$ Physical Examination:}

Examination begins, as it should for any injured patient, with the Advanced Trauma Life Support (ATLS) protocol of primary and secondary survey. Immediate attention may need to be directed to resuscitation and treatment of injuries that threaten life or limb. Knee evaluation is part of the secondary survey ${ }^{(\mathbf{1 9})}$.

Thetibial plateau fractures caused by highenergy mechanisms may be associated with neurological and vascular injury, compartment syndrome, deep vein thrombosis, contusion, crush injury to the soft tissues, or open wounds ${ }^{(\mathbf{1 9})}$. The evaluation and documentation of neurological status, vascular status, and soft tissues is essential in these fractures to plan for surgical treatment, and to decide on optimal timing of interventions ${ }^{(20)}$.

- Neurological examination focuses on the function of the peroneal nerve, which is the most vulnerable in the medial plateau fractures and high-energy fractures and is stretch on their way around the neck of the fibula. The tibial nerve is located in close proximity to the site of injury and also to be evaluated ${ }^{(20)}$.

- Vascular examination focuses on the popliteal artery, which can be injured by traction, debuting as a thrombosis, or may be sectioned by posteriorly displaced fracture fragments. If pulses are not palpable, and physical examination (capillary refill, color, and skin temperature) findings suggest vascular injury, use an echo Doppler. CT angiography (Fig. 1) and vascular surgical consultation should be performed ${ }^{(20)}$. 


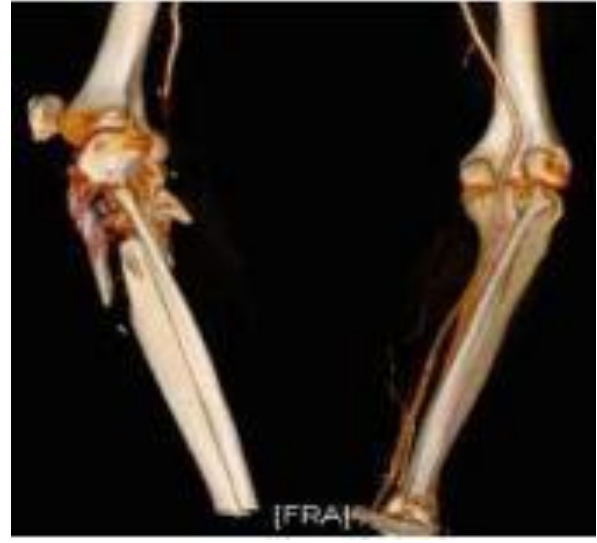

Figure (1): Angio-CT of complex fracture of the proximal tibia with stop in the popliteal artery ${ }^{(20)}$.

- The skin is inspected circumferentially;Contusion, blisters, and swelling are common in high-energy fractures (Fig.2) and should to be examined repeatedly ${ }^{(20)}$.

Open lesions should be excluded. We can inject methylene blue into the joint to assess whether there is communication between the joint and skin lacerations. The Gustilo-Andersonclassification is used for open fractures ${ }^{(20)}$.

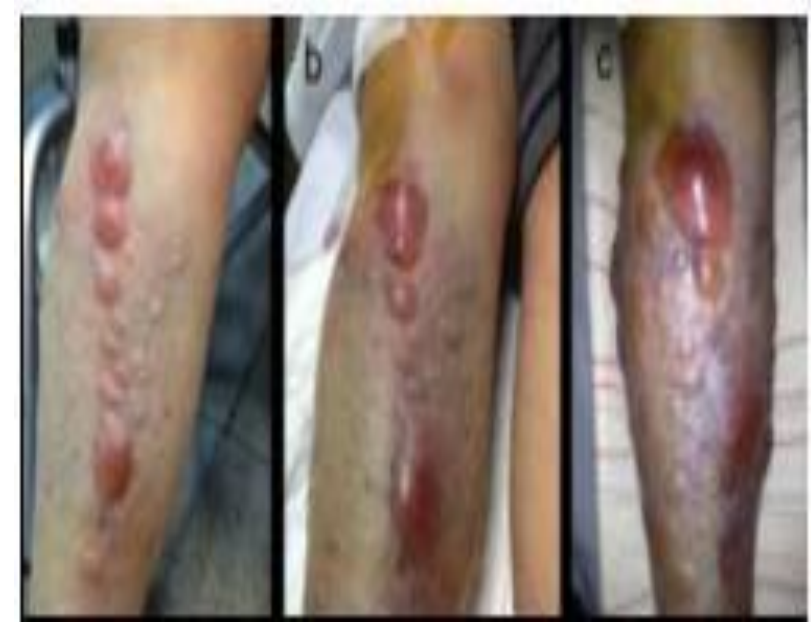

Figure (2): Evolution of soft-tissue status in tibial plateau fracture. (a) On arrival at the emergency department. (b) Three hours after trauma.(c) Six hours after trauma ${ }^{(20)}$.

- Ligament and stability testing should be done under anesthesia as it should to be delayed until the time of surgery to prevent the patient's pain and after bone stability. The instability should be compared with the opposite normal side. A fracture is considered stable if it does not exhibit, on varus or valgus stressing, any more than $10^{\circ}$ of instability at any point in the arc of motion, from full extension to $90^{\circ}$ of flexion ${ }^{(20)}$.

- The compartment syndrome is a terrible complication, diagnosis and early treatment is essential. The incidence of compartment syndrome in high- energy plateau fractures, especially types V and VI. (Corresponding three columns fracture $)^{(\mathbf{1 4})}$ is high measuring the pressure of the compartments (pressures within $30 \mathrm{mmHg}$ of the diastolic blood pressure) can be useful for diagnosis, but the clinical scene is prevalent (six Points: pain out of proportion, pain with passive stretch of muscle groups in the leg, pressure, paresthesias, paralysis, and pulselessness).The management must be urgent through fasciotomies of the four leg compartments ${ }^{(20)}$.

\section{$>$ Radiological assessment:}

No matter how thorough a history and physical examination is performed, diagnosis and operative planning cannot start without obtaining appropriate imaging ${ }^{(21)}$.

\section{- Radiography:}

Radiographic evaluation includes the standard knee trauma series of an anterior- posterior (AP), lateral and an AP view in the plane of the plateau (10-15 degree caudal view) are the standard examinations. Hohl ${ }^{(15)}$ found that the standard AP view could not reliably determine the amount of articular depression but that a 14-degree caudal view accurately estimated central and posterior displacement but could overestimate anterior displacement and depression. (Fig.3) ${ }^{(21)}$.

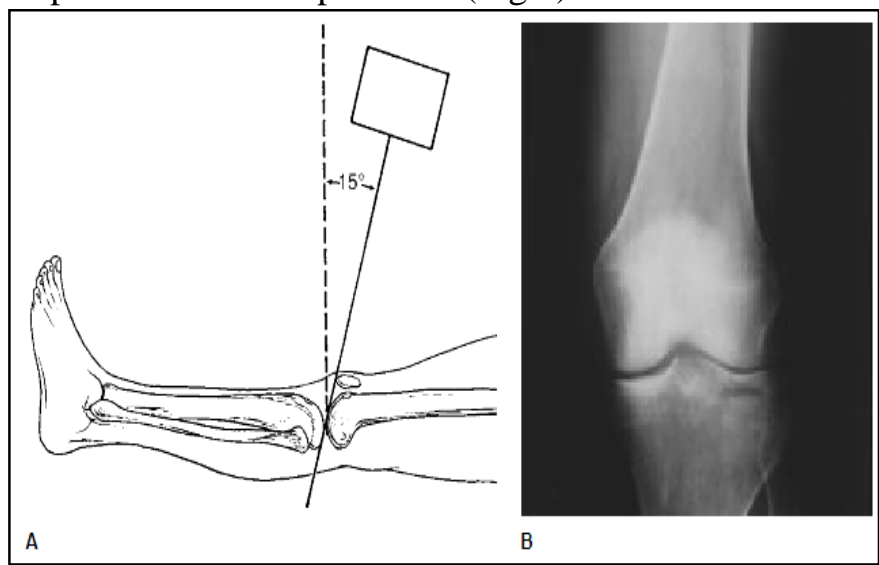

Figure (3): Schematic (A) and radiograph (B) depicting the 15-degree caudal radiographic view ${ }^{(21)}$.

Less frequently, oblique views are obtained to assess the location of fracture lines or degree of displacement but are not routine ${ }^{(\mathbf{2 1})}$.

\section{Computed Tomography Scans (C.T):}

The C.T scan is compelling for the precise diagnosis of tibial plateau fractures. Recently, several studies $^{(22,23)}$ concluded that a multiplanar CT scan and three dimensional reconstruction enhance the reliability in classifying tibial plateau fractures, as it can identify posterior column fractures and fragment likely to be missed on plain radiographs ${ }^{(23)}$.

The New Three-Column Classification is based on the information from CT scanning and 3-D reconstruction, which help the surgeon to have more accurate information for surgical planning ${ }^{(21)}$.

\section{- Magnetic Resonance Imaging:}

MRI assesses the location of fracture lines and the degree of articular displacement and also identifies occult fracture areas better than plain films and has been found to be equivalent to traditional two-dimensional C.T, MRI provides additional information about injuries to the soft tissue structures of the knee that is not 
obtained with other imaging modalities However, whether MRI should be a routine part of evaluating tibial plateau fractures or whether it should be used instead of C.T scanning is controversial ${ }^{(22)}$.

\section{Treatment of Comminuted Fracture of the Tibial Plateau:}

Goals of treatment of comminuted tibial plateau fractures include restoration of articular congruity, axial alignment, joint stability, and functional motion. If operative treatment is chosen, fixation must be stable enough to allow early motion and the technique should minimize wound complications ${ }^{(23)}$.

\section{* Timing of treatment:}

Open fractures with compartment syndrome and vascular injury must be treated immediately. However, patients with multiple injuries, especially those with head, thoracic, or abdominal injuries, must be stabilized provisionally using an external fixator (damage control orthopedics) until the general conditions improve ${ }^{(20)}$. On the other hand, closed fractures are challenging to manage, regarding the soft tissue injury. As severe crushing of the soft tissues may not be obvious at the beginning and therefore, careful observation of patients with closed fractures of the proximal tibia is mandatory ${ }^{(\mathbf{2 0})}$. Development of severe damage of soft tissues should not undergo open reduction and internal fixation immediately; in such cases it must stabilize the fracture provisionally by means of an external fixator performing indirect reduction via ligamentotaxis and restoration of limb length and covering fracture blisters with sterile non-adhesive dressings and regular monitoring of the compartments' pressure(Fig.4), elevation of the leg should follow and definitive surgical fracture fixation should be delayed until improvement of the soft tissue condition ${ }^{(20)}$.

The external fixator spans the knee joint with two femoral pins, proximal to the suprapatellar pouch, and two tibial pins. Tibial pins $(5 \times 170 \mathrm{~mm})$ must be implanted in the anteromedial surface, nearly perpendicular to the bone surface. Pins must be placed remote from anticipated skin incisions and anticipated implants. Femoral pins $(5 \times 170-200 \mathrm{~mm})$ can be placed anteriorly, laterally, or anterolaterally. Lateral pins avoid loss of knee motion due to scarring of the quadriceps, but the frame is mechanically inferior to that of anterior pins. The stability can improve by stacking the frame. The connector clamps must be placed outside of fracture to allow images of the reduced fracture without interference ${ }^{(20)}$.

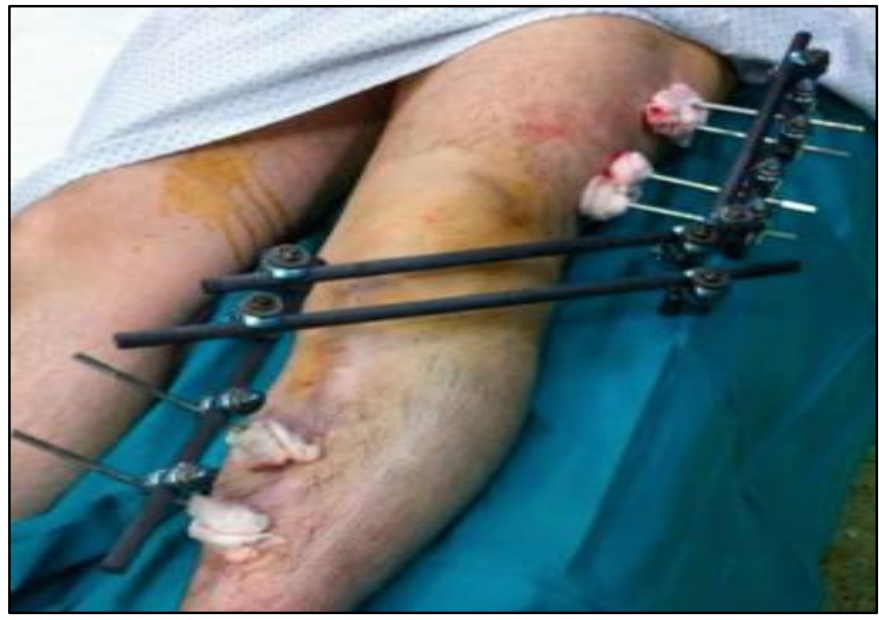

Figure (4): Clinical view of a temporary spanning external fixator ${ }^{(58)}$

\section{* Definitive treatment:}

\section{$>$ Conservative treatment:}

Nonoperative treatment for these high-energy injuries has a role in a medically decompensated patient only. These injuries have poor outcomes with nonoperative treatment ${ }^{(24)}$.

Conservative treatment is managed by using of a well molded long-leg cast, which is applied upon subsidence of the edema, with the knee in about $10^{\circ}$ of flexion and is maintained for 3-4 weeks, at this time the long cast can be replaced with a hinged brace and the patient can start mobilizing the knee joint, under supervision $^{(24)}$.

Initiation of weight-bearing depends on fracture configuration, patient's body mass and co-operation but, generally, touch weight-bearing can be allowed at 4-6 weeks and full weight-bearing at 8-12 weeks from the accident $^{(24)}$.

The goal of nonsurgical treatment is not anatomical reduction of the fracture but restoration of axial alignment and knee motion ${ }^{(24)}$.

\section{$>$ Operative Treatment:}

Because of the short proximal fragment and high incidence of comminution and/or significant intraarticular involvement, angular deformities and/or displacement occur frequently. In addition, the soft tissue envelope that surrounds the proximal tibia is tight and both closed and open fractures impose serious danger for the neurovascular structures that cross the specific anatomical area. For all these reasons, most proximal tibial fractures are not amenable to conservative management. Surgical treatment restores anatomy and allows early mobilization of the knee joint and weight-bearing of the limb ${ }^{(24)}$.

- Preoperative preparation and surgical technique:

Preoperative planning is essential for any complex injury; it forces the surgeon to understand the personality of the fracture and mentally prepare an operative strategy ${ }^{(25)}$. 
The definitive internal fixation in closed fractures should be performed when the soft tissues are improved (normally in 2 or 3 weeks). This can be evaluated by the decrease of inflammation, the perimeter of the leg, and the return of the ability of the skin to wrinkle $^{(25)}$.

The surgeon should understand the exact nature of the fracture before attempting any type of intervention. Radiographs of the contralateral extremity may benefit by serving as templates ${ }^{(25)}$.

All aspects of fracture reduction and fixation should be planned to avoid technical pitfalls. Ensure that all needed equipment is available, such as a tourniquet, a femoral distractor, osteotomies, bone tamp, suture anchors, bone graft substitutes, small and/or large fragment standard or periarticular plates and screws or external fixation devices of choice ${ }^{(25)}$.

The operative procedure should be performed under tourniquet on a radiolucent operating table with image intensification. Under general anesthesia, and after induction of antibiotic prophylaxis the procedure should be performed in the "floating position' based on a lateral decubitus, and the lower leg was rotated to a prone position when the posterior approach to the tibial plateau will be Performed (Fig. 5), this position could facilitate combined posterior and anterior approaches which is recommended by Zhu $\boldsymbol{e t} \boldsymbol{a l} .{ }^{(\mathbf{1 4})}$ for fixation of comminuted fracture of tibial plateau.

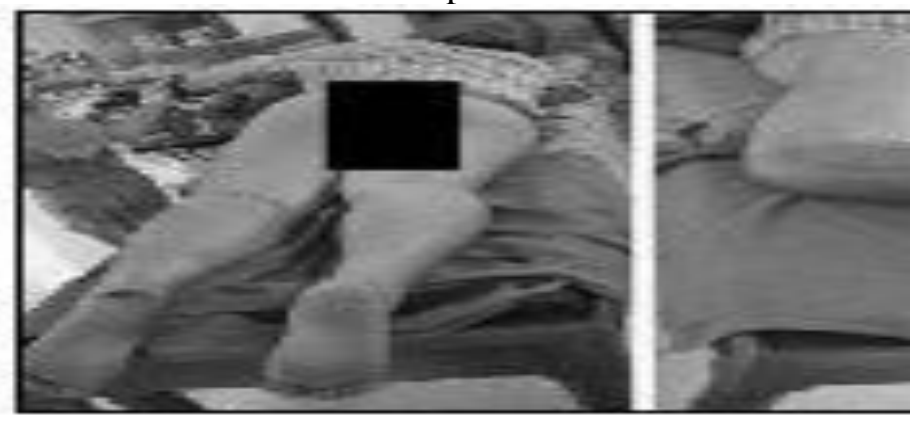

Figure (5): Combined approach: reversed L-shaped approach + anterior-lateral approach $^{(14)}$.

\section{> Postero-medial approach:}

A posterior inverted L-shaped approach was indicated to deal with medial column and posterior column fractures. With the patient prone on a radiolucent table, the knee was slightly flexed by a bump under the ankle. An inverted L-shaped incision begins at the center of popliteus parallel to Langers line superiorly and medial $^{(3)}$. Distally it turns at the medial corner of the popliteal fossa and is carried down to deep fascia (Fig.6a). Full-thickness fasciocutaneous flaps were raised paying attention to protecting the sural nerve and short saphenous vein. The tendon of the medial head of the gastrocnemius was then visualized (Fig.10-b) with blunt dissection and then retracted laterally, protecting the neurovascular bundle and displaying the back of the knee capsule (Fig. 6-c) ${ }^{(3)}$.

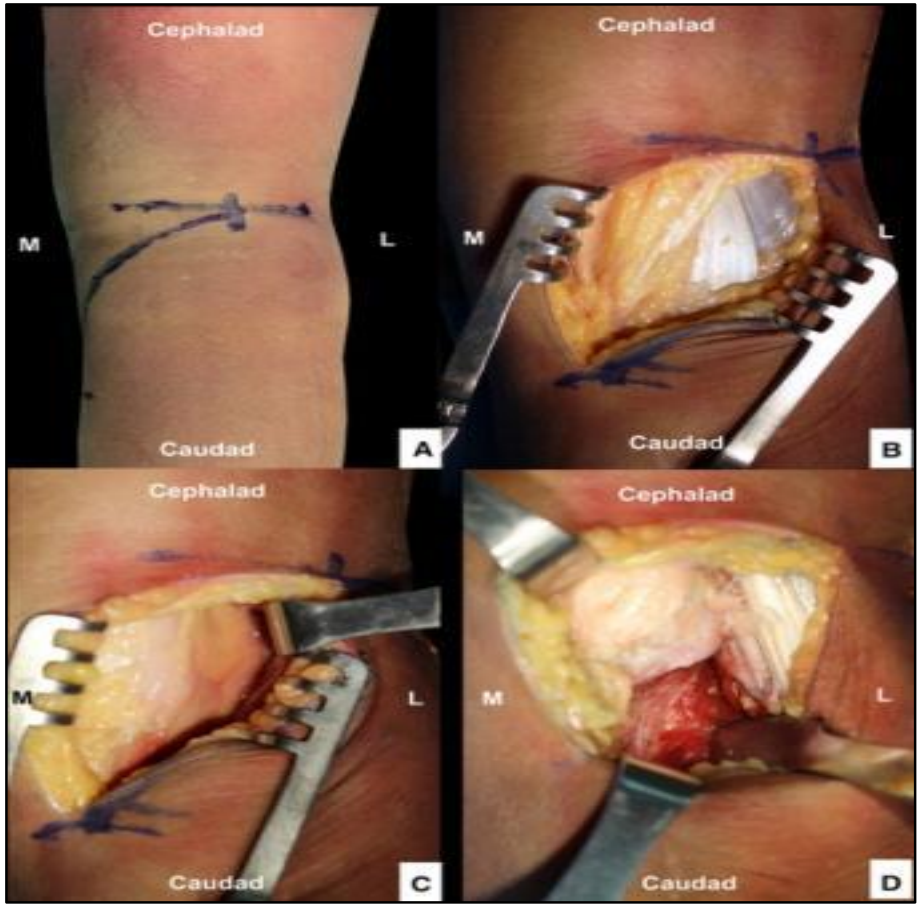

Figure (6): Reversed L posteromedial approach. A Reversed L incision. B and Cmedial gastrocnemius identified and retracted laterally. D Plane underneath the popliteus and soleus accessed ${ }^{(3)}$.

\section{Postoperative management and follow up:}

Early motion and non-weight bearing were emphasized in the postoperative protocols. Patients began passive knee range-of motion exercises from the first day post-operation. Weight bearing was started from toe-touch at eight weeks and increased progressively from eight to 12 weeks. Full weight bearing was not permitted until 12 weeks ${ }^{(26)}$ when the fracture was healed and callus appeared of radiographs ${ }^{(2)}$.

All patients undergo clinical observation and Xray film examination immediately after operation, at three, six and at 12 months post-operatively ${ }^{(2)}$.

Standard anterior-posterior and lateral radiographs are applied to determine bone-plate position, tibial plateau varus angle (TPA), posterior slope angle (PA) and fracture union progress. Functional and radiological outcomes are evaluated according to the modified Rasmussen score ${ }^{(\mathbf{2 6})}$. The functional scoring system comprises five parts: pain, walking capacity, extension, range of motion and stability; it ranges from 0 to 30 and can be graded as excellent, good, fair or poor. The radiological scoring system comprises three parts: depression, plateau width and deformity of the knee; it ranges from 0 to $10^{(26)}$.

\section{Complications of the tibial plateau fractures Loss of Reduction:}

High energy tibial plateau fractures have a high incidence of loss of reduction if not reduced and fixed properly. The clinical significance of loss of reduction is uncertain, but when it leads to malalignment, patient outcome may be compromised (Fig. 7). Improved 
methods of fixation and development of new implants may decrease the tendency for postoperative displacement ${ }^{(27)}$.

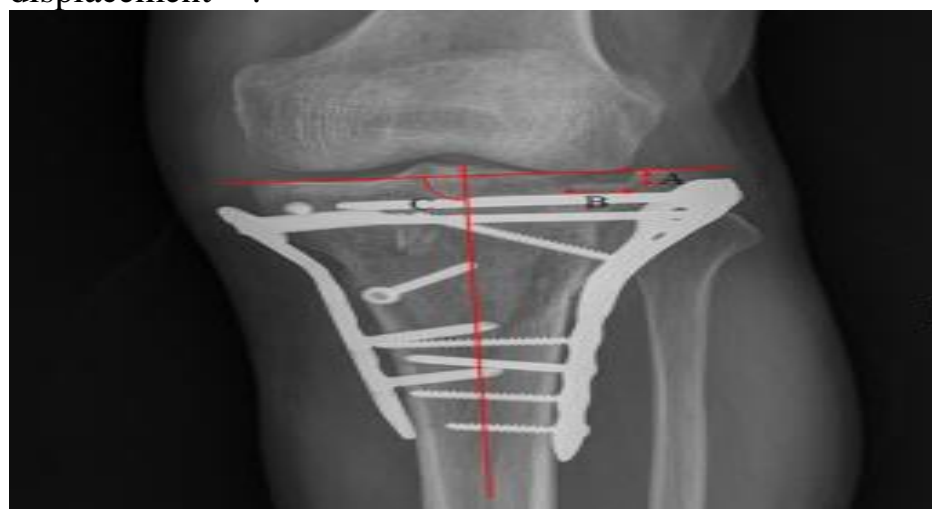

Figure (7): Measurement of the depression of articular surface, condylar widening, and alignment of the articular surface to the anatomical axis of tibia. (A) depression of articular surface, (B) condylar widening, and (C) alignment of the articular surface to the anatomical axis of tibia ${ }^{(27)}$.

\section{* Wound Breakdown and Infection:}

Wound-related problems, after operative treatment of tibia plateau fractures, are common and devastating complications that may lead to deep infection. Damage to the soft-tissue envelope, surgical incisions through contused skin and extensive dissection for the placement of implants often contribute to early wound breakdown and deep infection. Careful timing of surgery, extra periosteal dissection and limited dissection of comminuted bone fragments are likely to decrease complications ${ }^{(28)}$.

\section{* Deep venous thrombosis:}

The risk is exacerbated by the propensity for orthopedic patients to be bed bound for a number of days, preventing the 'calf pump' from moving blood out of the lower limbs and by the fact that orthopedic surgery on its own puts patients at increased risk ${ }^{(29)}$.

\section{* Neuro-vascular injury:}

The peroneal nerve is the most vulnerable in the medial plateau fractures and high-energy fractures and is stretch on their way around the neck of the fibula. The tibial nerve is located in close proximity to the site of injury and also to be evaluated ${ }^{(30)}$.

\section{* Knee stiffness or ankylosis:}

Limitation of knee motion after tibial plateau fractures is common. This complication is thought to result from damage to extensor retinaculum, to the joint surface as a consequence of the initial trauma or surgical exposure for fixation or to both. Extensor mechanism scarring with or without arthrofibrosis of the knee can lead to restricted knee movement. These effects are greatly magnified by immobilization after fracture or internal fixation. Early stable internal fixation of the fracture, meticulous soft-tissue handling and immediate mobilization of the knee maximize the chances of optimal outcomes after most tibial plateau fracture ${ }^{(28)}$.

\section{Malunion and nonunion:}

Malunion, with late articular collapse, or deformation of the metaphysis-shaft junction, can occur after operative treatment of tibia plateau fractures. Careful preoperative planning and stable internal fixation will reduce the incidence of these complications. If the mechanical load axis is affected, an osteotomy to restore the normal axis is indicated. If an articular surface malunion occurs in an older patient, a total-knee arthroplasty may be the best salvage procedure. In some circumstances, a loss of articular reduction occurs when a major joint fragment displaces. In these cases, early revision of the fixation should be considered, particularly if the displacement causes joint instability: late revision is extremely difficult ${ }^{(28)}$.

\section{- Prominent or Painful Hardware:}

Because the proximal tibia is largely subcutaneous, care must be taken to place implants in optimal positions to minimize prominent painful hardware ${ }^{(28)}$.

\section{* Implant Failure:}

Implant failures may occur due to intraoperative technical errors, including the use of plates that were too short and those that did not have adequate spanning segments (empty screw holes) over the fracture site and premature weight bearing before radiographic evidence of fracture healing ${ }^{(28)}$.

\section{Post-traumatic arthritis:}

Disruption of the articular surface of the knee can lead to gross, or subtle, alterations in knee kinematics ended by post-traumatic arthritis. Despite careful fracture reduction and fixation, residual symptoms are common. These range from mild aching with vigorous activities to progressively painful posttraumatic osteoarthritis ${ }^{(28)}$.

In the follow up; radiological narrowing of the joint space was noted during the first 7 years after injury, usually involving the same compartment as the fractured plateau, the incidence increased slightly with the age of the patients. Associated ligamentous injuries, as well as postoperative infection, increased the incidence of secondary degeneration and prompt to secondary osteoarthritis ${ }^{(28)}$.

\section{* Complications of bone grafting:}

Autogenous bone-graft harvesting is associated with significant morbidity. Reported minor complication includes cutaneous nerve damage, persistent discomfort, local wound complications including superficial wound infection, seroma and hematoma ${ }^{(\mathbf{3 1})}$. Autologous cancellous bone grafts from the iliac crest have a rather high morbidity, such as postoperative pain up to 2 years, difficulty in ambulation, hematoma and infection of the donor site as iliac crest abscess ${ }^{(\mathbf{3 1})}$. Allografts are associated with transmission of diseases like HIV and hepatitis ${ }^{(32)}$. 


\section{CONCLUSION}

The three-column classification of tibia plateau fractures based on reformatted 3D CT scans can identify posterior column fractures and fragment likely to be missed on plain radiographs. It used as a helpful tool for planning the surgical strategy. Using anatomical locking plates on tibial plateau is an effective and safe tool to treat complex three-column fractures of tibial plateau and is more convenient than common plate.

\section{REFERENCES}

1. Molenaars RJ, Mellema JJ, Doornberg JN et al. (2015): Tibial plateau fracture characteristics: computed tomography mapping of lateral, medial, and bicondylar fractures. JBJS., 97(18): 1512-1520.

2. Lin W, Su Y, Lin C et al. (2015): The application of a three-column internal fixation system with anatomical locking plates on comminuted fractures of the tibial plateau. International Orthopaedics, 264-015-2934-0.

3. Luo CF, Sun H, Zhang B et al. (2010): Three-column fixation for complex tibial plateau fractures. J Orthop Trauma, 24(11):683-692.

4. Prat-Fabregat S, Camacho-Carrasco $P$ (2016): Treatment strategy for tibial plateau fractures: an update. EFORT Open Reviews, 1(5), 225-232.

5. Barei DP, O'Mara TJ, Taitsman LA et al. (2008): Frequency and fracture morphology of the posteromedial fragment in bicondylar tibial plateau fracture patterns. J Orthop Trauma, 22:176-182.

6. Belen'kii IG, Iu Kochish A, Kislitsyn MA (2016): Fractures of the tibial condyles: current treatment methods and surgical approaches (literature review). Genij Ortopedii., 4: 114-122.

7. Joseph A (2005): Buckwalter. Articular cartilage and osteoarthritis. AAOS Instr Course Lect., 54:465-80.

8. Patrick E (2002): Meniscal Injury: I. Basic Science and Evaluation. J Am Acad Orthop Surg., 10(3):168-76.

9. Debbi EM, Wolf A, Goryachev Y et al. (2015): Alterations in sagittal plane knee kinetics in knee osteoarthritis using a biomechanical therapy device. Annals of Biomedical Engineering, 43(5): 1089-1097.

10. Thambyah A (2005): Contact stresses in the knee joint in deep flexion. Med Eng Phys., 27(4):329-35

11. Hashemi J, Chandrashekar N, Gill B et al. (2008): The geometry of the tibial plateau and its influence on the biomechanics of the tibiofemoral joint. The Journal of Bone and Joint Surgery. American Volume, 90(12): 2724-2729.

12. Francesc M, Jordi V, Pau G et al. (2015): Knee Anatomy and Biomechanics of the Knee. Sports Med Arthrosc Rev., 19(2):82-92.

13. Mortimer SL, Hunter R (2009): Arthroscopic Treatment of Knee Fractures. In: Brian P, McKeon, James V, Bono, John C, Richmond (Eds). Knee Arthroscopy, Tasto J P., (11):181-197.

14. Zhu Y, Hu CF, Yang G et al. (2013): Inter-observer reliability assessment of the Schatzker, AO/OTA and threecolumn classification of tibial plateau fractures." Journal of Trauma Management Outcomes, 7(1): 1-7.

15. Hohl M, Moore TM (1983): Articular fractures of the proximal tibia. Surgery of the musculoskeletal system. Available at: https://books.google.com.eg/ books?id $=$ GfITBQAAQBAJ\&pg
16. Schatzker J, McBroom R, Bruce D (1979): The tibial plateau fracture; The Toronto experience 1968 -1975. Clinical Orthopaedics and Related Research, 138: 94-104.

17. Brunner A, Horisberger M, Ulmar B et al. (2010): Classification systems for tibial plateau fractures; does computed tomography scanning improve their reliability? Injury, 41(2): 173-178.

18. Chen H, Shimin C, Jun $P$ (2015): Recent progress in the diagnosis and treatment of posterior tibial plateau fractures. International Journal of Clinical and Experimental Medicine, 8(4): 56-59.

19. Schatzker J, Watson JT, Levy B et al. (2009): Tibial plateau fractures. In: Browner, Jupiter, Levine, Trafton, editors. Skeletal Trauma, (4): 2074-2131.

20. Encinas-Ullán CA, Gómez-Cardero P, RodríguezMerchán EC (2014): Complex Fractures of the Proximal Tibia. In: Complex Fractures of the Limbs. Springer Cham., 14: 77-85.

21. Yoon RS, Frank AL, Kenneth AE (2015): Definitive Fixation of Tibial Plateau Fractures. Orthopedic Clinics of North America, 46(9): 363-375.

22. Marsh JL (2010): Tibial Plateau Fractures. In: Bucholz, Robert W.; Heckman, James D.; Court-Brown, Charles M.; Tornetta, Paul, Rockwood And Green's Fractures In Adults.Available at: https://www.ncbi.nlm. nih.gov/ pmc/articles/PMC3671120/

23. Matthew IR (2013): Fractures of the lower extremity. In: S. Terry Canale, James H. Beaty (eds.), Campbell's Operative Orthopedics, Pp. 2668-2679.

24. Garnavos C (2014): Management of Proximal Tibial Fractures. European Surgical Orthopedics and Traumatology, 16: 2825-2851.

25. Wiss D, Watson J, Johnson E (1996): Fractures of the Knee. In: Rockwood CA, Green DP, Bucholz RW, et al. (eds): Rockwood and Green's Fractures in Adults. Philadelphia: Lippincott-Raven, Pp. 1919-1999.

26. Rasmussen PS (1973): Tibial condylar fractures. Impairment of knee joint stability as an indication for surgical treatment. J Bone Joint Surg Am., 55(7):13311350 .

27. Ali AM, El-Shafie M, Willett M (2002): Failure of fixation of tibial plateau fractures. J Orthop Trauma, 16:323-329.

28. Papagelopoulos PJ, Partsinevelos AA, Themisto-cleous GS et al. (2006): Complications after tibia plateau fracture surgery. Injury, 37(6), 475-484.

29. Williams J, Hulstyn MJ, Fadale PD (1995): Incidence of deep vein thrombosis after arthroscopic knee surgery. A prospective study. Arthroscopy, 11:701-709.

30. Jöckel JA, Erhardt J, Vincenti M et al. (2014): Minimally invasive and open surgical treatment of proximal tibia fractures using a polyaxial locking plate system: a prospective multi-centre study. Int Orthop., 37(4):701-8.

31. Goulet JA (1997): Iliac crest bone graft: complications and functional assessment. Clin Orthop Relat Res., 339:76-81.

32. Segur JM, Torner $P$, García $S$ et al. (1998): Use of bone allograft in tibial plateau fractures. Orthop Trauma Surg., 117(6-7):357-359.

33. Wang Y, Luo C, Zhu Y et al. (2016): Updated threecolumn concept in surgical treatment for tibial plateau fractures-a prospective cohort study of 287 patients. Injury, 47(7): 1488-1496. 